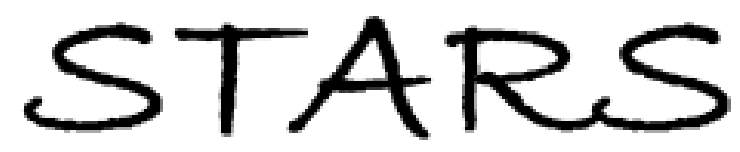

University of Central Florida

STARS

\title{
Effect of the concentration of organic dyes on their surface plasmon enhanced two-photon absorption cross section using activated Au nanoparticles
}

Ion Cohanoschi

University of Central Florida

Sheng Yao

University of Central Florida

Kevin D. Belfield

University of Central Florida

Florencio E. Hernández

University of Central Florida

Find similar works at: https://stars.library.ucf.edu/facultybib2000

University of Central Florida Libraries http://library.ucf.edu

This Article is brought to you for free and open access by the Faculty Bibliography at STARS. It has been accepted for inclusion in Faculty Bibliography 2000s by an authorized administrator of STARS. For more information, please contactSTARS@ucf.edu.

\section{Recommended Citation}

Cohanoschi, Ion; Yao, Sheng; Belfield, Kevin D.; and Hernández, Florencio E., "Effect of the concentration of organic dyes on their surface plasmon enhanced two-photon absorption cross section using activated $\mathrm{Au}$ nanoparticles" (2007). Faculty Bibliography 2000s. 6968.

https://stars.library.ucf.edu/facultybib2000/6968

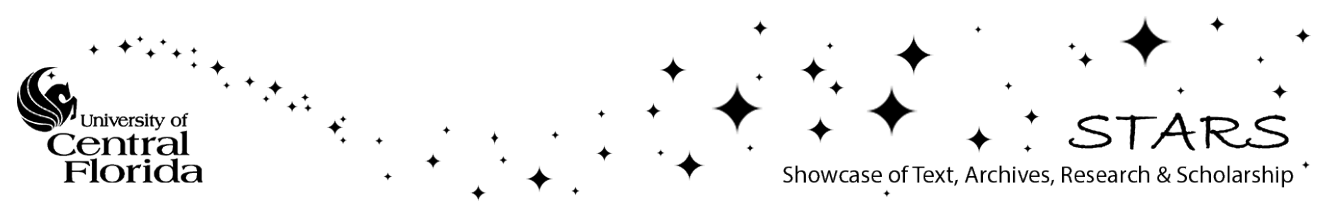




\section{Effect of the concentration of organic dyes on their surface plasmon enhanced two- photon absorption cross section using activated Au nanoparticles}

Cite as: J. Appl. Phys. 101, 086112 (2007); https://doi.org/10.1063/1.2718897

Submitted: 18 December 2006 . Accepted: 21 February 2007 . Published Online: 30 April 2007

Ion Cohanoschi, Sheng Yao, Kevin D. Belfield, and Florencio E. Hernández

\section{ARTICLES YOU MAY BE INTERESTED IN}

Two-photon excited surface plasmon enhanced energy transfer between DAPI and gold nanoparticles: Opportunities in intra-cellular imaging and sensing

Applied Physics Letters 99, 103701 (2011); https://doi.org/10.1063/1.3633066

Electromagnetic fields around silver nanoparticles and dimers

The Journal of Chemical Physics 120, 357 (2004); https://doi.org/10.1063/1.1629280

Vibronic coupling simulations for linear and nonlinear optical processes: Theory

The Journal of Chemical Physics 136, 06411 (2012); https://doi.org/10.1063/1.3684236

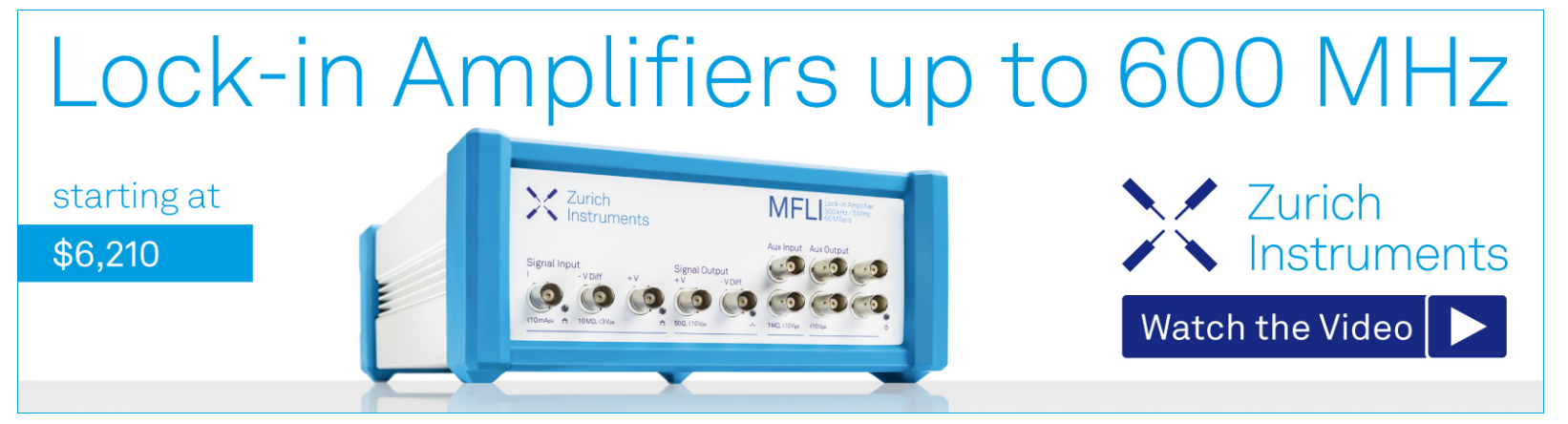




\title{
Effect of the concentration of organic dyes on their surface plasmon enhanced two-photon absorption cross section using activated Au nanoparticles
}

\author{
Ion Cohanoschi, Sheng Yao, ${ }^{a}$ Kevin D. Belfield, and Florencio E. Hernández ${ }^{\text {b) }}$ \\ Department of Chemistry and College of Optics \& Photonics/CREOL \& FPCE, \\ University of Central Florida, P.O. Box 162366, Orlando, Florida 32816-2366
}

(Received 18 December 2006; accepted 21 February 2007; published online 30 April 2007)

\begin{abstract}
In this article we present the study of the surface plasmon enhanced two-photon absorption of a hydrophilic stilbene derivative (trans-4,4'-diaminostilbene) in aqueous solution at different concentrations. The observed exponential growth of the effective two-photon absorption cross section $\left[\sigma_{2}^{\prime}(\mathrm{Au})\right]$ is attributed to the electric-field augmentation via surface plasmon resonance between nanoparticles, i.e., hot spots, and the molecular density on $\mathrm{Au}$ nanospheres. An unprecedented $\sigma_{2}^{\prime}(\mathrm{Au})=550000 \mathrm{GM}$ has been measured. This result opens a new universe of applications in multiphoton imaging, photodynamic therapy, telecommunications, optical limiting, and multidimensional data storage using hybrid systems. (C) 2007 American Institute of Physics.
\end{abstract}

[DOI: $10.1063 / 1.2718897]$

Two-photon absorption (2PA) processes are based on the simultaneous absorption of two photons at a wavelength that is two times longer than that corresponding to single-photon excitation. ${ }^{1}$ The excitation probability for 2PA scales quadratically with the incident irradiance, confining it to the immediate vicinity of the focal plane of a focused beam. Therefore, the spatial resolution is considerably increased ${ }^{2}$ and, because linear absorption at the excitation wavelengths is negligible, the penetration depth is much greater. ${ }^{3}$ As a result, 2PA has found many applications in confocal imaging, ${ }^{4-6}$ and photodynamic therapy (PDT), ${ }^{7-13}$ among others. Nevertheless, for concrete applications in the biomedical field, there is still a gap to fulfill on the specific design of molecules that exhibit stronger 2PA, and the development of alternative processes that can augment the effective 2PA cross section of organic molecules by reducing the irradiance requirement. The latter can be achieved by means of the electric-field enhancement produced by the surface plasmon on metal nanoparticles. ${ }^{14}$

The tremendous electric-field enhancement observed at the surface plasmon resonance (SPR) has been applied to nonlinear optical processes such as second-harmonic generation (SHG), ${ }^{15,16}$ Raman scattering, ${ }^{17,18}$ amplification of light, ${ }^{19}$ and more recently to two-photon absorption (2PA). ${ }^{20-24}$ Surface plasmon enhanced fluorescence has been demonstrated through two-photon induced fluorescence in a Kretschmann geometry ${ }^{21,23}$ and, near-field fluorescence microscopy using metal tips. ${ }^{22,24}$ Enhancements greater than two orders of magnitudes have been reported. Also, a fluorescence enhancement factor as high as $10^{5}$ via $2 \mathrm{PA}$ has been demonstrated on thiol containing dyes, attached to silver nanoparticle fractal clusters. ${ }^{25}$ The extraordinary increase has been attributed to the strong electric-field enhancement ef-

\footnotetext{
a) Only with the Department of Chemistry.

b) Author to whom all correspondence should be addressed. Electronic address: florenzi@mail.ucf.edu
}

fects in localized "hot spots." More recently, we reported a 480-fold enhancement of the effective two-photon absorption cross section of Hoechst 33258 in an aqueous solution containing activated gold colloid. ${ }^{26}$

In order to have a better understanding of the SPR assisted 2PA in organic molecules, here we present the study of the effect of the concentration of a hydrophilic stilbene derivative, trans-4,4'-diaminostilbene (SY183) in aqueous solution, in the presence of activated gold nanoparticles.

Gold nanospheres of $12 \mathrm{~nm}$ average diameter $(<15 \%$ size distribution) were synthesized using the well-known Turkevich et al. $^{27,28} 50 \mathrm{~mL}$ of preboiled $2.5 \times 10^{-4} \mathrm{M}$ $\mathrm{AuHCl}_{4}$ aqueous solution were mixed with $1.0 \mathrm{~mL}$ of $3.4 \times 10^{-3} \mathrm{M}$ sodium citrate solution and, heated for $5 \mathrm{~min}$. The deep red color of the solution revealed the presence of gold nanoparticles. ${ }^{27,29}$ A typical SPR band with maximum at approximately $520 \mathrm{~nm}$ was observed using the Agilent 8453 spectrophotometer (see Fig. 1). The nanoparticles size and size distribution were determined by transmission electron microscopy using a FEI Tecnai F30 TEM (images not included). In order to ensure 2PA enhancement using longer excitation wavelengths in the NIR region, it was essential to activate the metal colloids by electrolyte-induced aggregation, just as in surface enhanced Raman scattering (SERS). ${ }^{26,30-32}$ Activated gold colloid suspensions (hot particles) were prepared by adding $0.5 \mathrm{~mL}$ of $1 \mathrm{M} \mathrm{NaCl}(a q)$ to $5.0 \mathrm{~mL}$ of the original gold nanoparticle solution. The presence of optically active hot particles was confirmed by uv-vis absorption spectroscopy. As a result of the coupling of the individual dipole oscillators of small isolated particles, the uv-vis absorption spectrum turned into a typical broad absorption band of activated gold colloid that extended to at least $850 \mathrm{~nm}$ (see Fig. 1). ${ }^{26,32}$

Dye-hot particles samples were prepared by mixing different volumes (0.085-1.32 mL) of a $1.65 \times 10^{-2}$ M SY $183 / \mathrm{H}_{2} \mathrm{O}$ with $5.5 \mathrm{~mL}$ of the activated gold colloidal solution. The final concentration of SY183 in the activated 


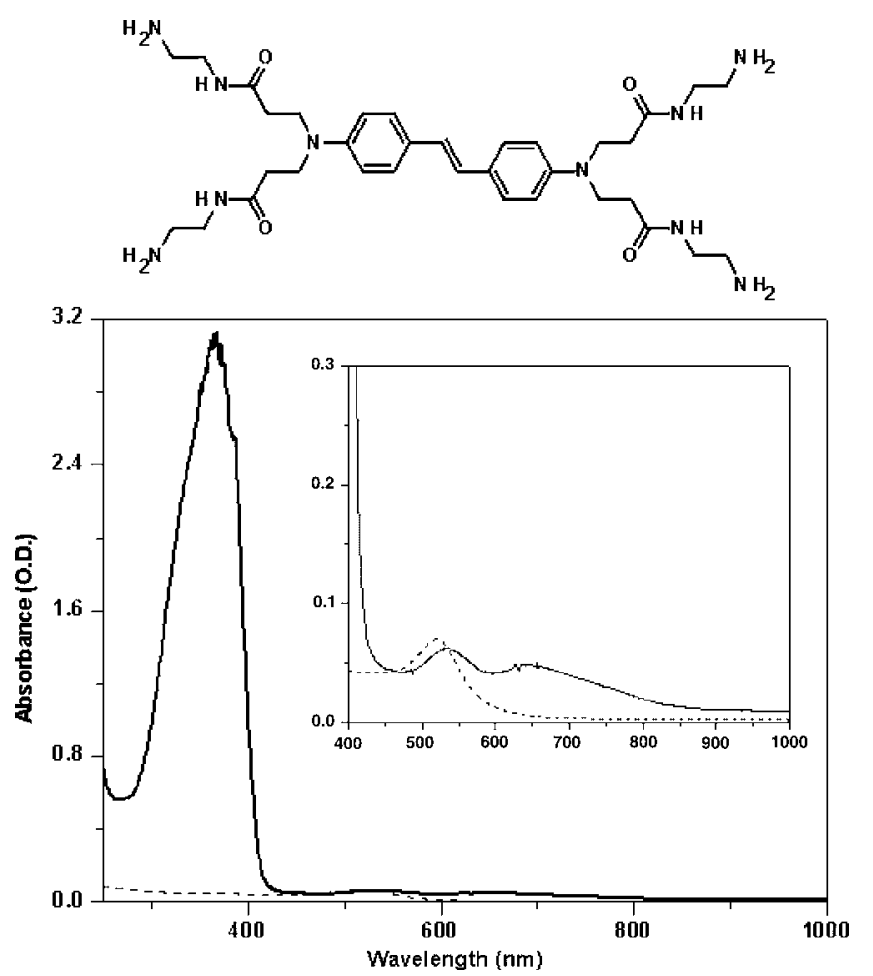

FIG. 1. Molecular structure of trans-4,4'-diaminostilbene (top). Absorption spectra of SY183/ $\mathrm{H}_{2} \mathrm{O}$ solution $\left(10^{-3} \mathrm{M}\right)$ in the presence of activated gold colloid (solid line) and, pure Au nanoparticles in solution (dashed line). The inset shows a closer view of the low absorbance region of the spectra.

gold containing solutions was between $2.5 \times 10^{-4}$ and 3.2 $\times 10^{-3} \mathrm{M}$. The molecular structure of SY183 as well as the absorption spectrum of the final mixture at $3.2 \times 10^{-3} \mathrm{M}$ is shown in Fig. 1. The dye in the presence of hot particles did not change its absorption spectrum, indicating no structural changes. The SPR band of hot particles in activated gold solution was shifted to approximately $535 \mathrm{~nm}$. This suggested that dye molecules were partially recovering the hot particles. It is well known that the position of the SPR band is very sensitive to the dielectric constant of the surrounding medium $\varepsilon_{m}{ }^{14}$ Because SY183 presents a higher $\varepsilon_{m}$ than water, as dye molecules recover the metal surface, a redshift of the SPR band is expected. ${ }^{14}$ Measurements of pure dye were done on $\mathrm{NaCl}(a q)$ containing $\mathrm{SY} 183 / \mathrm{H}_{2} \mathrm{O}$ solution at a final concentration $1 \times 10^{-3} \mathrm{M}$. $\mathrm{NaCl}(a q)$ was added to this solution to avoid matrix effects.

For the determination of the effective two-photon absorption cross section of SY183 in the presence of Au nanoparticles $\sigma_{2}^{\prime}(\mathrm{Au})$, we employed the well-known open aperture $Z$-scan method. ${ }^{33}$ For this purpose we used an EKSPLA 401 OPG system pumped with a mode-locked, 25 ps EKSPLA PL-2143B laser [full width at half maximum (FWHM)] and, working at a $10 \mathrm{~Hz}$ repetition rate. The maximum two-photon excitation was induced at $600 \mathrm{~nm}$, the wavelength corresponding to the highest $2 \mathrm{PA}$ cross section and to the $S_{0} \rightarrow S_{2}$ transition, for SY183. ${ }^{34}$

In Fig. 2 we show the experimental values of the 2PA cross-sectional enhancement factor $\left\lfloor\sigma_{2}^{\prime}(\mathrm{Au}) / \sigma_{2}\right\rfloor$ versus SY183 concentration (solid squares), where $\sigma_{2}=3500 \mathrm{GM} .^{34}$ An exponential growing of $\sigma_{2}^{\prime}(\mathrm{Au})$ with the dye concentration was found. In order to explain the experimental obser-

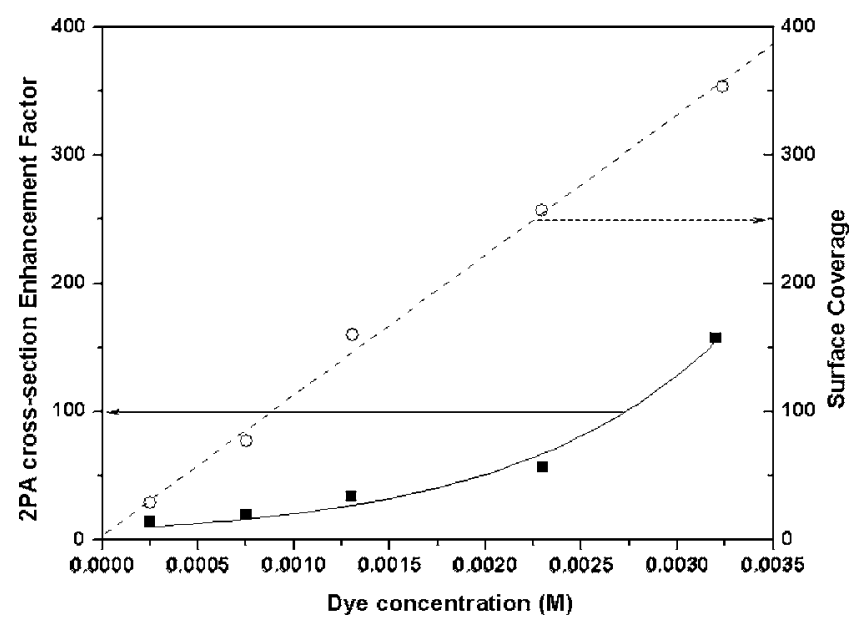

FIG. 2. The effective 2PA cross section of trans-4,4'-diaminostilbene as function of dye concentration (solid square). The solid line represents the theoretical fitting using an exponential function. The number of SY183 molecules per gold nanosphere (surface coverage) vs dye concentration (empty circles). The dashed line represents the theoretical linear fitting.

vation, the surface molecular density of SY183 on Au nanoparticles was estimated by the approach described below. However, the fact that for this calculation, cluster formation was not considered should be first highlighted. Although, this seems to be a rough approximation because particle clusters formation was intentionally induced to perform the experiments there is no way one can know exactly the average cluster size in solution. Nevertheless, considering that only those molecules placed at hot spots can experience the electric-field enhancement, this approximation can still be appropriate to qualitatively explain the experimental results.

The surface area $S_{g}=452 \mathrm{~nm}^{2}$, of spherical gold nanoparticles with $12 \mathrm{~nm}$ average diameter was calculated first. Then, the maximum number of molecules that can be attached to an Au nanoparticle were estimated considering the dye molecules as small cylinders of $1.3 \mathrm{~nm}$ in diameter and, attached radially to the gold nanospheres surface. The diameter of the molecules was determined by adding up bond distances across the molecular axis on the molecular plane. The radial attachment was deduced based on the molecular geometry and the amino groups at its extremities (see Fig. 1), which can be electrostatically bound to conductive metal surfaces. ${ }^{35}$ Based on these assumptions the maximum number of dye molecules that can bind to the surface of one metal nanosphere is $348\left(S_{g} / 0.25 \pi 1.3^{2}\right)$.

Next, we estimated the molecular density for each SY183 concentration in the presence of $\mathrm{Au}$ nanoparticles. The number of gold atoms contained within one nanosphere was calculated knowing that a gold unit cell contains four atoms in a CCF structure with $407.82 \mathrm{pm}$ lattice size. This yields a unit cell volume $V_{u}=0.068 \mathrm{~nm}^{3}$. Because the volume $(V)$ of one $12 \mathrm{~nm}$ diameter nanosphere is $905 \mathrm{~nm}^{3}$, one gold nanoparticle contains $5.3 \times 10^{4}$ atoms $\left(D=4 \times V / V_{u}\right)$. The number of gold nanoparticles present in solution was determined knowing the initial concentration of $\mathrm{HAuCl}_{4} \mathrm{em}-$ ployed for the synthesis of the gold nanoparticles using the Turkevich method, and considering a reaction with a $100 \%$ yield. Based on the reduction of $50 \mathrm{~mL}$ of $\mathrm{HAuCl}_{4}$ 
$\left(2.5 \times 10^{-4} \mathrm{M}\right)$ by trisodium citrate, the total number of gold atoms in solution was $7.5 \times 10^{18}$. Because only $5 \mathrm{~mL}$ of the final nanoparticle suspension were used, the total number of gold atoms to be considered were $7.5 \times 10^{17}$. The ratio $7.5 \times 10^{17} / D$ yielded a total number of nanoparticles $N_{P}=1.4 \times 10^{13}$. In order to determine the actual number of dye molecules attached to all gold nanospheres in suspensions, we used a centrifugation method to separate the excess of SY183 dissolved in solution from the dye/nanoparticle composite. All five samples were centrifuged for $30 \mathrm{~min}$ at 4000 rpm using a Vari Hi-Speed Centricone equipment. The number of dye molecules in solution before $\left(N_{d 1}\right)$ and after centrifugation $\left(N_{d 2}\right)$ were determined measuring their absorbance and using Beer's law:

$$
\begin{aligned}
& N_{d 1}=N_{A} \times d_{1} \times V_{s}=5.4207 \times 10^{20} \times d_{1}, \\
& N_{d 2}=N_{A} \times d_{2} \times V_{s}=5.4207 \times 10^{20} \times d_{2} .
\end{aligned}
$$

Here, $N_{A}$ is the Avogadro's number, $d_{1}$ and $d_{2}$, are the dye concentration before and after centrifugation, respectively, and $V_{s}$ is the sample volume. The dye molecules attached to the gold nanoparticles $N_{F}$ was given by the difference between $N_{d 1}$ and $N_{d 2}$ :

$$
N_{F}=N_{d 1}-N_{d 2}=5.4207 \times 10^{20}\left(d_{1}-d_{2}\right) .
$$

The number of dye molecules attached to the surface of one nanosphere $R$ was obtained by dividing $N_{F}$ to the total number of gold nanoparticles within the sample volume $\left(N_{P}\right)$ :

$$
R=N_{F} / N_{P}=3.213 \times 10^{8}\left(d_{1}-d_{2}\right) .
$$

The plot of $R$ versus concentration is shown in Fig. 2 (empty circles). It is obvious that as the concentration of SY183 increases the surface coverage also increases, thus, $\sigma_{2}^{\prime}(\mathrm{Au})$ increases. Because the maximum SY183 concentration used in the experiments turned out to be equal to the highest amount of dye that can be dissolved in water and the maximum $\mathrm{Au}$ nanoparticle surface coverage, the greatest achievable enhancement of the 2PA cross section of SY183 is $550000 \mathrm{GM}$. Knowing that the 2PA cross section of pure SY183 is $\sigma_{2}^{\prime}=3500 \mathrm{GM},{ }^{34}$ the maximum effective $2 \mathrm{PA}$ crosssectional enhancement for this particular molecule is 157 . Because of solubility issues, it was impossible to increase the dye concentration in solution any further to find the expected saturation region where the enhancement comes to an end. Supplementary studies will be performed.

At this point an interesting question to ask is if the observed enhancement in the 2PA is due to the electric-field augmentation at the SPR or, if it is perhaps due to the greater number of molecules at the surface of the nanoparticles? If it is just the result of the molecular density, the 2PA should change directly proportional with the dye concentration and not exponentially as it has been shown in Fig. 2. In addition, the number of molecules within the focusing volume should be the same for the same initial SY183 concentration in the absence and in the presence of nanoparticles. Therefore, the enhanced 2PA is the result of the electric-field augmentation.

In summary, it has been demonstrated that metal surface coverage has a crucial effect on the surface plasmon enhanced 2PA of organic dyes attached to, or in the immediate vicinity of metal nanoparticles. The $2 \mathrm{PA}$ enhancement is due to the electric-field enhancement via SPR at the surface of activated gold nanoparticles colloidal solutions. In addition, a maximum effective enhancement of the effective twophoton absorption cross section of 157 -fold for SY183 has been demonstrated.

This research was supported by start-up funds provided to FEH by the Department of Chemistry, University of Central Florida, and the In-House Research Award (No. 11649003), UCF.

${ }^{1}$ M. Goeppert-Mayer, Ann. Phys. 9, 273 (1931).

${ }^{2}$ W. Denk, J. H. Strickler, and W. W. Webb, Science 248, 73 (1990).

${ }^{3}$ R. W. Boyd, Nonlinear Optics (Academic, San Diego, CA, 1992), Chap.

4, p. 8.

${ }^{4}$ D. R. Larson, W. R. Zipfel, R. M. Williams, S. W. Clark, M. P. Bruchez, F. W. Wise, and W. W. Webb, Science 300, 1434 (2003).

${ }^{5}$ J. M. Squirrel, D. L. Wokosin, J. G. White, and B. D. Bavister, Nat. Biotechnol. 17, 763 (1999).

${ }^{6}$ R. H. Kohler, J. Cao, W. R. Zipfel, W. W. Webb, and M. R. Hansen, Science 276, 2039 (1997).

${ }^{7}$ A. Karotki, M. A. Drobizhev, M. Kurk, A. Rebane, E. Nickel, and C. W. Spangler, Proc. SPIE 4612, 143 (2002).

${ }^{8}$ S. J. Madsen, C. Sun, B. J. Tomberg, V. P. Wallace, and H. Hirschberg, Photochem. Photobiol. 72, 128 (2000).

${ }^{9}$ E. A. Wachter, W. P. Partridge, W. G. Fisher, H. C. Dees, and M. G. Petersen, Proc. SPIE, 68 (1998).

${ }^{10}$ P. K. Frederiksen, M. Jorgensen, and P. R. Ogilby, J. Am. Chem. Soc. 123, 1215 (2001).

${ }^{11}$ W. G. Fisher, W. P. Partridge, C. Dees, and E. Wachter, Photochem. Photobiol. 66, 141 (1997).

${ }^{12}$ T. D. Poulsen, P. K. Frederiksen, M. Jorgensen, K. V. Mikkelsen, and P. R. Ogilby, J. Phys. Chem. 105, 11488 (2001).

${ }^{13}$ D. Bhawalkar, N. D. Kumar, C. F. Zhao, and P. N. Prasad, J. Clin. Laser Med. Surg. 15, 201 (1997).

${ }^{14}$ H. Raether, Surface Plasmons on Smooth and Rough Surfaces and Grattings (Springer-Verlag, New York, 1988).

${ }^{15}$ C. K. Chen, A. R. B. de Castro, and Y. R. Chen, Phys. Rev. Lett. 46, 145 (1981).

${ }^{16}$ A. Wokaun, J. G. Bergman, J. P. Heritage, A. M. Glass, P. F. Liao, and D. H. Olson, Phys. Rev. B 24, 849 (1981).

${ }^{17}$ A. Otto, I. Mrozek, H. Grabhorn, and W. Akemann, J. Phys.: Condens. Matter 4, 1143 (1992).

${ }^{18}$ S. Nie and S. R. Emory, Science 275, 1102 (1997).

${ }^{19}$ J. R. Lakowicz, Anal. Biochem. 298, 1 (2001).

${ }^{20}$ H. Kano and S. Kawata, Opt. Lett. 21, 1848 (1996).

${ }^{21}$ E. Z. Kretschmann, J. Appl. Phys. 241, 313 (1971).

${ }^{22}$ E. J. Sanchez, L. Novotny, and X. S. Xie, Phys. Rev. Lett. 82, 4014 (1999).

${ }^{23}$ I. Gryczynski, J. Malicka, Y. Shen, Z. Gryczynski, and J. R. Lakowicz, J. Phys. Chem. B 106, 2191 (2002).

${ }^{24}$ Y. Shen, J. Swiatkiewicz, T.-C. Lin, P. Markowicz, and P. N. Prasad, J. Phys. Chem. B 106, 4040 (2002).

${ }^{25}$ W. Wenseleers, F. Stellacci, T. Meyer-Friedrichsen, T. Mangel, C. A. Bauer, S. J. K. Pond, S. R. Marder, and J. W. Perry, J. Phys. Chem. B 106, 6853 (2002).

${ }^{26}$ I. Cohanoschi and F. E. Hernandez, J. Phys. Chem. B 109, 14506 (2005).

${ }^{27}$ J. Turkevich, P. C. Stevenson, and J. Hiller, Discuss. Faraday Soc. 11, 55 (1951).

${ }^{28}$ G. Frens, Nat. Phys. 241, 20 (1973).

${ }^{29}$ J. R. Lakowicz, Principles of Fluorescence Spectroscopy, 2nd ed. (Kluwer Academic, New York, 1999).

${ }^{30}$ G. Schatz, Acc. Chem. Res. 17, 370 (1984).

${ }^{31}$ M. Moskovits, Rev. Mod. Phys. 57, 783 (1985).

${ }^{32}$ V. M. Shalaev, Phys. Rep. 272, 61 (1996).

${ }^{33}$ M. Sheik-Bahae, A. A. Said, T. Wei, D. J. Hagan, and E. W. Van Stryland, IEEE J. Quantum Electron. 26, 760 (1990).

${ }^{34}$ S. Yao, K. J. Schafer-Hales, I. Cohanoschi, F. E. Hernandez, and K. D. Belfield, Synlett 12, 1863 (2006).

${ }^{35}$ K. G. Thomas and P. V. Kamat, Acc. Chem. Res. 36, 888 (2003). 